\title{
Aiding and opposing mixed convection of water with density inversion about a wall of varying temperature
}

\author{
RAJENDRA PRASAD SONI and MADHUSUDHANA R GAVARA* \\ Department of Mechanical Engineering, Indian Institute of Technology Guwahati, Guwahati 781039, India \\ e-mail: gmadhu@iitg.ac.in
}

MS received 27 November 2018; revised 13 April 2019; accepted 17 April 2019; published online 2 July 2019

\begin{abstract}
Mixed convection of water over a vertical surface of varying temperature with density inversion is studied for aiding and opposing convection configurations. The temperature of the surface is assumed to be an arbitrary function of vertical distance. The governing equations are transformed using dimensionless stream function and temperature. The temperature differentials of varying wall temperature are used as perturbation functions. The dimensionless stream function and temperature are expanded in power series of perturbation variables and coefficient functions. The obtained coefficient functions are valid for any arbitrary wall temperature function, and hence they are 'universal functions'. Power law wall temperature variation is chosen to show the usefulness of universal functions. The results are presented for velocity and temperature distributions in the boundary layer, velocity and thermal boundary layer thicknesses, skin friction coefficient and heat transfer rates for various values of governing parameter and wall temperature power law index for both aiding and opposing flows. It is found that for the range of $G r_{y} / R e_{y}^{2}$ values considered in the study, the skin friction coefficient and heat transfer rates vary almost linearly with wall temperature power law index value for a given $G r_{y} / R e_{y}^{2}$ value for both aiding and opposing flows. For special wall temperature cases, the present results are compared to benchmark solutions available in literature and good agreement is found.
\end{abstract}

Keywords. Mixed convection; non-isothermal vertical plate; density inversion of water; aiding and opposing convection.

\section{Introduction}

Mixed convection heat transfer has numerous applications in industries as well as in nature. The applications include flows of atmosphere and oceans, electronics cooling, solar energy collectors, heat exchangers, thermal design of buildings, etc. The applications of convective flows with density inversion of water can be found in many systems such as in thermal energy storage system using ice for cooling and thermal processing, refrigeration and air-conditioning process, atmospheric flows, oceanic movements, etc. Usually density of fluid monotonically decreases with respect to temperature. However, density of water shows increasing and decreasing trend with temperature, in a certain temperature range, near $4^{\circ} \mathrm{C}$, showing a local density maximum. The increased density with temperature is referred to as density inversion. This unusual nature of water introduces peculiarities in buoyancy forces. Hence, mixed convection, which is influenced by buoyancy forces, shows peculiarities in this temperature range. This drives us

*For correspondence to study the effect of density inversion of water in mixed convection flows.

Many authors studied the mixed convection flow over a vertical plate with different wall thermal conditions. Study of mixed convection flow over isothermally heated vertical surface for various Prandtl numbers was carried out in [1, 2]. Lloyd and Sparrow [1] used local similarity method for mixed convection flows, for various values of Richardson number and found that the method is not accurate for large values of Richardson number studied in the Prandtl number range. However, Acrivos [2] theoretically investigated the combined effect of free and forced convection using asymptotic solution for various Prandtl numbers. The author reported that mixed convection depends on both Richardson and Prandtl numbers when Prandtl number is greater than 1 , while the convection depends on Richardson number only, for lower Prandtl number. Szewczyk [3] presented a series solution for aiding and opposing mixed convection flow for a few Prandtl numbers. The results were demonstrated using velocity and temperature distributions, shear stress and heat transfer for various values of Richardson number. Hussain and Afzal [4] numerically investigated aiding and opposing 
mixed convection flow over a vertical plate for uniform wall temperature/heat flux conditions. The authors implemented a computer extension perturbation series method and predicted results up to three-digit accuracy for skin friction coefficient and heat transfer rates. The obtained results were compared to available numerical results and good agreement was found. Lin and Chen [5] numerically studied aiding and opposing mixed convection over an isothermal vertical plate for a wide range of Prandtl number using Keller's box method. They proposed a new mixed convection parameter, which is valid for any Prandtl number value, to replace the usual mixed convection parameter (Richardson number).

Both free and forced convection from an isothermal vertical moving plate were numerically examined for a wide range of Prandtl number by Lin and Hoh [6]. The plate moved either in streamwise or in opposite to streamwise direction. They presented correlations for heat transfer in terms of Prandtl number. Raju et al [7] investigated mixed convection boundary layer flow over vertical and horizontal isothermal surfaces for aiding and opposing flow situations. The authors found flow separation points in opposing flows and obtained the relationship for local Nusselt number with Prandtl number. Chen et al [8] studied the effect of Prandtl number on mixed convection flow for various plate orientations. Correlations were obtained for local and average Nusselt number for both aiding and opposing flow situations. The results were compared with experimental and numerical results.

The effect of variable wall temperature on mixed convection boundary layer flow was studied by a few researchers. Merkin and Pop [9] numerically studied the effect of small and large Prandtl numbers on mixed convection flow over a vertical surface for various values of mixed convection parameter. The surface temperature of plate was inversely proportional to the distance from leading edge. It was found that unique solutions exist for all considered mixed convection parameters in case of aiding flow, while dual solutions were observed for opposing flow. Saeid [10] numerically studied the effect of surface temperature variation on mixed convection flow for air and water. The author analysed the convection for varying wall temperature with periodic oscillations in time and constant wall temperature. It was reported that Nusselt number and skin friction coefficient showed periodical variations for different amplitudes and frequencies of temperature for both aiding and opposing flows. Numerical investigation of mixed convection boundary layer flow in a stable stratified medium adjacent to a vertical surface for various Prandtl numbers was carried out by Ishak et al [11], using Keller's box method. The wall temperature is a linear function of vertical distance. They found the existence of dual solutions and boundary layer separation due to thermal stratification for assisting flow.

Several experimental results were reported for mixed convection flows over a vertical plate. An experimental investigation of combined forced and free convection boundary layer flow over a vertical isothermal surface was carried out by Gryzagoridis [12]. The velocity and temperature profiles, obtained using hot wire anemometry, were presented for various governing parameters. The calculated heat transfer rates were compared to theoretical results available in literature. Kobus and Wedekind [13] analysed development of thermal boundary layer along a vertical isothermal surface by introducing a special model. Special closed-form solutions for local and average Nusselt numbers were presented for various Prandtl numbers for both aiding and opposing mixed convection. An experimental study was also performed and experimental results matched well with theoretical results available in literature. An experiment was conducted for mixed convection flows over an isothermally heated vertical plate by Venugopal et al [14]. A correlation for Nusselt number was presented and good agreement was found between experimental results and available numerical results.

The density inversion of water brings significant effect on mixed convection flows. Goren [15] analysed free convection flow of water near its density inversion about a vertical plate. The author presented results for temperature and velocity distributions. He found that for a small temperature difference, at maximum density, convective currents become weak compared with those of water without density inversion. Gebhart et al [16] numerically examined buoyancy-induced flows adjacent to a horizontal surface with water density inversion for constant wall temperature and heat flux conditions. They presented solutions for various values of wall temperature variation parameter. They found sudden decreases in heat transfer rate near the density inversion point. Vighnesam and Soundalgekar [17] studied combined effect of free and forced convection flow of water near its density inversion about a vertical plate with power law varying wall temperature using similarity solutions. The effects of various values of $G r_{y} / R e_{y}^{2}$ and power law index were studied for both aiding and opposing flows. They reported that Nusselt number increases with increasing value of power law index for both aiding and opposing flows. However, skin friction coefficient decreases with power law index values in aiding flow, while it increases in opposing flow situation.

The effect of porous medium on mixed convection is an area of interest for a few authors. In an analysis, Kumaran and Pop [18] theoretically studied the free convection boundary layer flow over a vertical plate in a porous medium with water near its density inversion. They studied the effect of wall temperature and heat flux on natural convection, which are power law functions of vertical distance from leading edge of the plate. They derived the similarity form of boundary layer equations and presented their numerical and analytical results for velocity and temperature distributions and heat transfer rates. The effect of viscosity of fluid varying with temperature on mixed 
convection flow in a porous medium over an isothermal vertical surface was studied by Chin et al [19]. They studied the effect of mixed convection and variable viscosity parameters on fluid flow and heat transfer characteristics. They observed dual solutions and boundary layer separations in case of opposing flows.

Several mathematical techniques are used in the literature to solve the boundary layer convective flows. For the solutions of boundary layer flows, similarity methods of $[1,9]$, approximate solutions such as local non-similarity method of $[20,21]$ and series expansion method of [22] were used.

Perturbation method has been used to solve boundary layer flows by a few authors such as Yang and Jerger [23] and Seetharamu and Dutta [24], due to its inherent advantages. In a recent study, Gavara et al [25] used perturbation method to solve the boundary layer mixed convection over a vertical plate in air with temperature boundary condition. In the present study, boundary layer mixed convection of water flow with the effect of density inversion for arbitrarily varying wall temperature is solved using perturbation method. The main advantage of perturbation method is that the calculated universal functions are valid for an arbitrary wall temperature variation. Hence, the results can be easily used to get solutions for any arbitrary wall temperature variation condition without the need for solving any differential equation again for a different temperature variation.

\section{Physical system}

The physical system of the present study is shown in figure 1 . Water flows over the vertical surface and forms the mixed convection boundary layer. The free-stream velocity is $v_{\infty}$ and free-stream temperature of the water is $T_{0}$, which

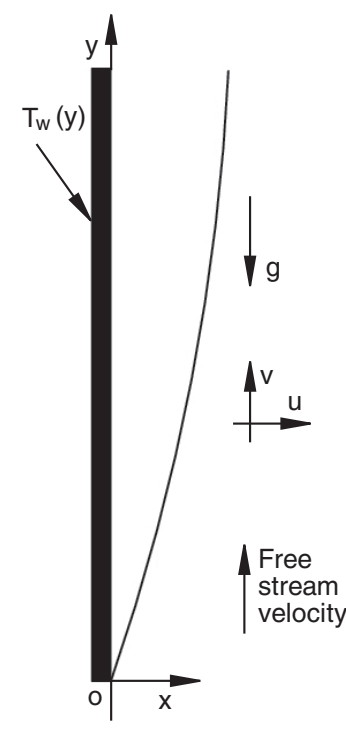

(a) Aiding mixed convection (b) Opposing mixed convection

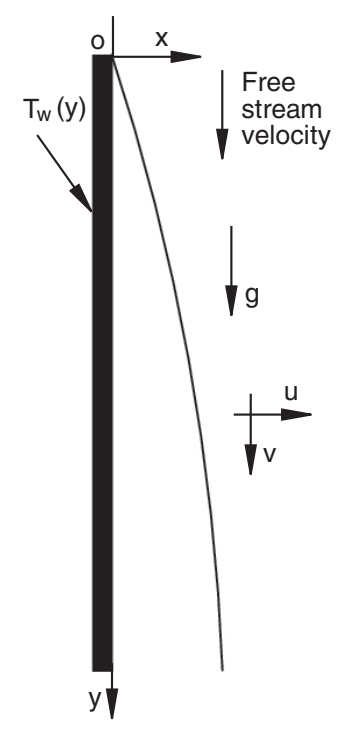

Figure 1. Schematic of physical and coordinate systems. is the temperature corresponding to density maximum $\left(\rho_{0}\right)$. The temperatures of the wall and water are considered in the range for which the density inversion of water is valid. The plate is subjected to an arbitrarily varying wall temperature that is a function of vertical distance. Both the aiding as well as opposing mixed convection are solved in this study. The physical and coordinate systems are shown in figure 1(a) and 1(b) for aiding and opposing mixed convection, respectively.

\section{Mathematical model}

A steady, laminar and incompressible flow is assumed. The effects of viscous dissipation and radiation on mixed convection are assumed to be negligible. The study is carried out for cases where the boundary layer approximations are valid. The temperature in the boundary layer is assumed to be in the range for which water shows density inversion. The properties of water are assumed to be constant, except the density variation with respect to temperature in buoyancy term. The variation of density with respect to temperature is approximated using different functions by various authors of $[16,17,26]$ in the literature. In the present study, the following density-temperature approximation is considered, which is used in [17]:

$$
\frac{\rho}{\rho_{0}}=1-\gamma\left(T-T_{0}\right)^{2}
$$

where $\rho_{0}$ is the maximum density at temperature $T_{0}=3.98^{\circ} \mathrm{C}$ and $\gamma=8.0 \times 10^{-6}\left({ }^{\circ} \mathrm{C}\right)^{-2}$ is a constant. This correlation is valid for the temperature range $0-12^{\circ} \mathrm{C}$ with admissible error limit that is reported in [26]. Under these assumptions, the governing equations for mixed convection flows can be written as follows.

Continuity equation

$$
\frac{\partial u}{\partial x}+\frac{\partial v}{\partial y}=0
$$

$y$-momentum equation

$$
u \frac{\partial v}{\partial x}+v \frac{\partial v}{\partial y}=v \frac{\partial^{2} v}{\partial x^{2}} \pm g \gamma\left(T-T_{0}\right)^{2}
$$

Energy equation

$$
u \frac{\partial T}{\partial x}+v \frac{\partial T}{\partial y}=\alpha \frac{\partial^{2} T}{\partial x^{2}} .
$$

The ' + ' and '-' signs in Eq. (3) refer to aiding and opposing mixed convection flows, respectively.

No slip and no penetration conditions are specified at the wall. The free-stream velocity and temperature are uniform, and $v_{\infty}$ and $T_{0}$, respectively. Mathematically, the boundary conditions are as follows: 


$$
\begin{gathered}
\text { at } \quad x=0: \quad u=v=0, \quad T=T_{w}(y) ; \\
\text { at } \quad x \rightarrow \infty: \quad v \rightarrow v_{\infty}, \quad T \rightarrow T_{0} .
\end{gathered}
$$

To eliminate the coupling between continuity and momentum equations, $u$ and $v$ velocities are expressed in terms of stream function as

$$
u=-\frac{\partial \psi}{\partial y}, \quad v=\frac{\partial \psi}{\partial x} .
$$

With these $u$ and $v$ velocities definitions, the momentum and energy equations given by Eqs. (3) and (4), respectively, are converted into

$$
\begin{gathered}
\frac{\partial \psi}{\partial x} \frac{\partial^{2} \psi}{\partial x \partial y}-\frac{\partial \psi}{\partial y} \frac{\partial^{2} \psi}{\partial x^{2}}=v \frac{\partial^{3} \psi}{\partial x^{3}} \pm g \gamma\left(T-T_{0}\right)^{2} \\
\frac{\partial \psi}{\partial x} \frac{\partial T}{\partial y}-\frac{\partial \psi}{\partial y} \frac{\partial T}{\partial x}=\alpha \frac{\partial^{2} T}{\partial x^{2}}
\end{gathered}
$$

The boundary conditions (5) and (6) in stream function forms are as follows:

$$
\begin{gathered}
\text { at } \quad x=0: \quad \frac{\partial \psi}{\partial x}=\frac{\partial \psi}{\partial y}=0, \quad T=T_{w}(y) ; \\
\quad \text { at } \quad x \rightarrow \infty: \quad \frac{\partial \psi}{\partial x} \rightarrow v_{\infty}, \quad T \rightarrow T_{0} .
\end{gathered}
$$

\section{Solution of the governing equations}

For the solutions of the governing equations, the following transformations are applied in terms of dimensionless stream function $f$, dimensionless temperature $\theta$ and independent variable $\eta$ :

$$
\begin{gathered}
f\left(\eta, \lambda_{0}, \lambda_{1}, \ldots, \lambda_{n}, \ldots\right)=\frac{\psi(x, y)}{\sqrt{v v_{\infty} y}}, \\
\theta\left(\eta, \lambda_{0}, \lambda_{1}, \ldots, \lambda_{n}, \ldots\right)=\frac{T(x, y)-T_{0}}{T_{w}(y)-T_{0}}, \\
\eta(x, y)=x \sqrt{\frac{v_{\infty}}{v y}} .
\end{gathered}
$$

In these transformations, $\lambda_{0}, \lambda_{1}, \ldots, \lambda_{n}$ are a set of perturbation variables, which are functions of vertical distance $y$. It can be observed that these transformations are similar to the transformations by similarity variable method except that in these transformations, perturbation variables are present additionally.

Using the transformations given by Eqs. (12)-(14), the momentum and energy equations (8) and (9), respectively, are transformed into the following forms:

$$
\begin{gathered}
\frac{\partial^{3} f}{\partial \eta^{3}}=-\frac{f}{2} \frac{\partial^{2} f}{\partial \eta^{2}} \pm \frac{G r_{y}}{R e_{y}^{2}} \theta^{2}-\frac{\partial^{2} f}{\partial \eta^{2}} y \sum_{n=0}^{\infty} \frac{\partial f}{\partial \lambda_{n}} \frac{\partial \lambda_{n}}{\partial y} \\
+\frac{\partial f}{\partial \eta} y \sum_{n=0}^{\infty} \frac{\partial^{2} f}{\partial \lambda_{n} \partial \eta} \frac{\partial \lambda_{n}}{\partial y}, \\
\frac{1}{\operatorname{Pr}} \frac{\partial^{2} \theta}{\partial \eta^{2}}=-\frac{\partial f}{\partial \eta} y \sum_{n=0}^{\infty} \frac{\partial \theta}{\partial \lambda_{n}} \frac{\partial \lambda_{n}}{\partial y}+\frac{\partial \theta}{\partial \eta} y \sum_{n=0}^{\infty} \frac{\partial f}{\partial \lambda_{n}} \frac{\partial \lambda_{n}}{\partial y}-\frac{f}{2} \frac{\partial \theta}{\partial \eta} \\
+y \frac{\partial f}{\partial \eta}\left(\frac{\theta}{T_{w}-T_{0}}\right) \frac{\partial T_{w}}{\partial y} .
\end{gathered}
$$

In these equations, $G r_{y} / R e_{y}^{2}$ is the governing parameter, which is a local modified Richardson number. $G r_{y}$ and $R e_{y}$ are modified Grashof number and Reynolds number, respectively, which are

$$
G r_{y}=\frac{g \gamma\left(T_{w}-T_{0}\right)^{2} y^{3}}{v^{2}}
$$

and

$$
\operatorname{Re}_{y}=\frac{v_{\infty} y}{v} .
$$

The boundary conditions (10) and (11) in terms of dimensionless parameters $f, \theta$ and $\eta$ are as follows:

$$
\begin{gathered}
\text { at } \quad \eta=0: \quad f=0, \quad \frac{\partial f}{\partial \eta}=0, \quad \theta=1 ; \\
\text { at } \quad \eta \rightarrow \infty: \quad \frac{\partial f}{\partial \eta} \rightarrow 1, \quad \theta \rightarrow 0 .
\end{gathered}
$$

It is seen that Eqs. (15) and (16) involve two independent variables $y$ and $\eta$ and the equations are in the form of partial differential equations. It can also be observed from the analysis that Eqs. (15) and (16) become functions of $\eta$ and $\lambda_{n}$ if $\lambda_{n}(y)$ is taken in the following form [24]:

$$
\lambda_{n}(y)=\frac{y^{n+1}}{T_{w}-T_{0}} \frac{d^{n+1}}{d y^{n+1}}\left(T_{w}-T_{0}\right)
$$

where $T_{w}-T_{0}$ is infinitely differentiable with respect to $y$. It is found that even with this definition of $\lambda_{n}(y)$, the resulting equations contain independent variables $\eta$ and $\lambda_{n}(y)$ and the equations are still partial differential equations. To convert these equations into sets of ordinary differential equations, the dimensionless stream function and dimensionless temperature are expanded in the following forms:

$$
\begin{gathered}
f\left(\eta, \lambda_{0}, \lambda_{1}, \ldots, \lambda_{n}, \ldots\right)=F(\eta)+\lambda_{0} f_{0}(\eta)+\lambda_{1} f_{1}(\eta)+\lambda_{2} f_{2}(\eta) \\
+\cdots+\lambda_{0}^{2} f_{00}(\eta)+\lambda_{1}^{2} f_{11}(\eta)+\cdots
\end{gathered}
$$




$$
\begin{aligned}
& \theta\left(\eta, \lambda_{0}, \lambda_{1}, \ldots, \lambda_{n}, \ldots\right)=H(\eta)+\lambda_{0} \theta_{0}(\eta)+\lambda_{1} \theta_{1}(\eta) \\
& +\lambda_{2} \theta_{2}(\eta)+\cdots+\lambda_{0}^{2} \theta_{00}(\eta)+\lambda_{1}^{2} \theta_{11}(\eta)+\cdots
\end{aligned}
$$

where $\lambda_{0}, \lambda_{1}, \ldots, \lambda_{n}(y), \ldots$ are the perturbation variables while $F(\eta), f_{0}(\eta), f_{1}(\eta), \ldots, f_{00}(\eta), \ldots$ and $H(\eta), \theta_{0}(\eta)$, $\theta_{1}(\eta), \ldots, \theta_{00}(\eta), \ldots$ are coefficient functions for stream function and temperature, respectively. Substituting Eqs. (21)-(23) in Eqs. (15) and (16), the equations take the following forms:

$$
\begin{aligned}
F^{\prime \prime \prime} & +\lambda_{0} f_{0}^{\prime \prime \prime}+\lambda_{1} f_{1}^{\prime \prime \prime}+\lambda_{2} f_{2}^{\prime \prime \prime}+\cdots+\lambda_{0}^{2} f_{00}^{\prime \prime \prime}+\lambda_{1}^{2} f_{11}^{\prime \prime \prime}+\cdots= \\
& -\frac{1}{2} F F^{\prime \prime} \pm \frac{G r_{y}}{R e_{y}^{2}} H^{2}+\lambda_{0}\left(-\frac{1}{2} F f_{0}^{\prime \prime}-\frac{3}{2} f_{0} F^{\prime \prime}+F^{\prime} f_{0}^{\prime}\right. \\
& \left.\frac{G r_{y}}{R e_{y}^{2}} 2 H \theta_{0}\right)+\lambda_{1}\left(-\frac{1}{2} F f_{1}^{\prime \prime}-\frac{5}{2} f_{1} F^{\prime \prime} \pm \frac{G r_{y}}{R e_{y}^{2}} 2 H \theta_{1}\right. \\
& \left.-F^{\prime \prime} f_{0}+F^{\prime} f_{0}+2 F^{\prime} f_{1}^{\prime}\right)+\lambda_{2}\left(-\frac{1}{2} F f_{2}^{\prime \prime}-\frac{7}{2} f_{2} F^{\prime \prime}\right. \\
\pm & \left.\frac{G r_{y}}{R e_{y}^{2}} 2 H \theta_{2}-F^{\prime \prime} f_{1}+F^{\prime} f_{1}^{\prime}+3 F^{\prime} f_{2}^{\prime}\right)+\lambda_{0}^{2}\left(-\frac{1}{2} F f_{00}^{\prime \prime}\right. \\
& -\frac{5}{2} f_{00} F^{\prime \prime}-\frac{3}{2} f_{0} f_{0}^{\prime \prime} \pm \frac{G r_{y}}{R e_{y}^{2}}\left(H \theta_{00}+2 H \theta_{0}\right)+F^{\prime \prime} f_{0} \\
+ & \left.2 F^{\prime} F_{00}^{\prime}+f_{0}^{\prime} f_{0}^{\prime}-F^{\prime} f_{0}^{\prime}\right)+\lambda_{1}^{2}\left(-\frac{1}{2} F f_{11}^{\prime \prime}-\frac{5}{2} F^{\prime \prime} f_{11}\right. \\
& -\frac{3}{2} f_{1} f_{1}^{\prime \prime}-f_{0} f_{1}^{\prime \prime}+2 F^{\prime} F_{11}^{\prime} \pm \frac{G r_{y}}{R e_{y}^{2}}\left(\theta_{1}^{2}+2 H \theta_{11}\right) \\
+ & \left.f_{0}^{\prime} f_{1}^{\prime}+2 f_{1}^{\prime} f_{1}^{\prime}\right)+\cdots,
\end{aligned}
$$

$$
\begin{aligned}
\frac{1}{P r}\left(H^{\prime \prime}\right. & \left.+\lambda_{0} \theta_{0}^{\prime \prime}+\lambda_{1} \theta_{1}^{\prime \prime}+\lambda_{2} \theta_{2}^{\prime \prime}+\cdots+\lambda_{0}^{2} \theta_{00}^{\prime \prime}+\lambda_{1}^{2} \theta_{11}^{\prime \prime}+\cdots\right)= \\
& -\frac{1}{2} F H^{\prime}+\lambda_{0}\left(-\frac{1}{2} F \theta_{0}^{\prime}-\frac{3}{2} f_{0} H^{\prime}+F^{\prime} \theta_{0}+H F^{\prime}\right) \\
& +\lambda_{1}\left(-\frac{1}{2} F \theta_{1}^{\prime}-\frac{5}{2} f_{1} H^{\prime}+F^{\prime} \theta_{0}+2 F^{\prime} \theta_{1}-H^{\prime} f_{0}\right) \\
& +\lambda_{2}\left(-\frac{1}{2} F \theta_{2}^{\prime}-\frac{7}{2} f_{2} H^{\prime}+F^{\prime} \theta_{1}+3 F^{\prime} \theta_{2}-H^{\prime} f_{1}\right) \\
& +\lambda_{0}^{2}\left(-\frac{1}{2} F \theta_{00}^{\prime}-\frac{5}{2} f_{00} H^{\prime}+2 F^{\prime} \theta_{00}-\frac{3}{2} f_{0} \theta_{0}^{\prime}+f_{0}^{\prime} \theta_{0}\right. \\
& \left.+H^{\prime} f_{0}+H f_{0}\right)+\lambda_{1}^{2}\left(-\frac{1}{2} F \theta_{11}^{\prime}-\frac{9}{2} f_{11} H^{\prime}+4 F^{\prime} \theta_{11}\right. \\
& \left.-\frac{5}{2} f_{1} \theta_{1}^{\prime}+f_{0}^{\prime} \theta_{0}+2 f_{1}^{\prime} \theta_{1}-f_{0} \theta_{1}^{\prime}\right)+\cdots
\end{aligned}
$$

Note that in these equations, primes $\left({ }^{\prime}\right)$ denote differentiation with respect to $\eta$.

Equating of coefficients such as $\lambda_{0}, \lambda_{1}, \lambda_{2}, \ldots, \lambda_{n}(y), \ldots$ of these ordinary differential equations on either side, we get infinite number of sets of ordinary differential equations of various universal functions. Larger the number of sets of equations considered in the calculation, larger the accuracy of the solutions $[27,28]$. The change in the solutions is found to be negligible if the sets of equations are considered beyond $\lambda_{0}, \lambda_{1}, \lambda_{2}, \lambda_{0}^{2}$ and $\lambda_{1}^{2}$. The sets of equations considered in the present study are as follows.

Constant term:

$$
\begin{aligned}
F^{\prime \prime \prime} & =-\frac{1}{2} F F^{\prime \prime} \pm \frac{G r_{y}}{R e_{y}^{2}} H^{2}, \\
\frac{1}{P r} H^{\prime \prime} & =-\frac{1}{2} F H^{\prime} .
\end{aligned}
$$

The set of coefficient of $\lambda_{0}$ :

$$
\begin{aligned}
f_{0}^{\prime \prime \prime} & =-\frac{1}{2} F f_{0}^{\prime \prime}-\frac{3}{2} f_{0} F^{\prime \prime} \pm \frac{G r_{y}}{R e_{y}^{2}} 2 H \theta_{0}+F^{\prime} f_{0}^{\prime}, \\
\frac{1}{P r} \theta_{0}^{\prime \prime} & =-\frac{1}{2} F \theta_{0}^{\prime}-\frac{3}{2} f_{0} H^{\prime}+F^{\prime} \theta_{0}+H F^{\prime} .
\end{aligned}
$$

The set of coefficient of $\lambda_{1}$ :

$$
\begin{aligned}
f_{1}^{\prime \prime \prime}= & -\frac{1}{2} F f_{1}^{\prime \prime}-\frac{5}{2} f_{1} F^{\prime \prime} \pm \frac{G r_{y}}{R e_{y}^{2}} 2 H \theta_{1}-F^{\prime \prime} f_{0} \\
& +F^{\prime} f_{0}+2 F^{\prime} f_{1}^{\prime}, \\
\frac{1}{P r} \theta_{1}^{\prime \prime}= & -\frac{1}{2} F \theta_{1}^{\prime}-\frac{5}{2} f_{1} H^{\prime}+F^{\prime} \theta_{0}+2 F^{\prime} \theta_{1}-H^{\prime} f_{0} .
\end{aligned}
$$

The set of coefficient of $\lambda_{2}$ :

$$
\begin{aligned}
f_{2}^{\prime \prime \prime}= & -\frac{1}{2} F f_{2}^{\prime \prime}-\frac{7}{2} f_{2} F^{\prime \prime} \pm \frac{G r_{y}}{R e_{y}^{2}} 2 H \theta_{2}-F^{\prime \prime} f_{1} \\
& +F^{\prime} f_{1}^{\prime}+3 F^{\prime} f_{2}^{\prime}, \\
\frac{1}{P r} \theta_{2}^{\prime \prime}= & -\frac{1}{2} F \theta_{2}^{\prime}-\frac{7}{2} f_{2} H^{\prime}+F^{\prime} \theta_{1}+3 F^{\prime} \theta_{2}-H^{\prime} f_{1} .
\end{aligned}
$$

The set of coefficient of $\lambda_{0}^{2}$ :

$$
\begin{aligned}
f_{00}^{\prime \prime \prime} & =-\frac{1}{2} F f_{00}^{\prime \prime}-\frac{5}{2} f_{00} F^{\prime \prime}-\frac{3}{2} f_{0} f_{0}^{\prime \prime}+F^{\prime \prime} f_{0}+f_{0}^{\prime} f_{0}^{\prime} \\
& +2 F^{\prime} F_{00}^{\prime}-F^{\prime} f_{0}^{\prime} \pm \frac{G r_{y}}{R e_{y}^{2}}\left(\theta_{0}^{2}+2 H \theta_{00}\right), \\
\frac{1}{P r} \theta_{00}^{\prime \prime} & =-\frac{1}{2} F \theta_{00}^{\prime}-\frac{5}{2} f_{00} H^{\prime}+2 F^{\prime} \theta_{00}-\frac{3}{2} f_{0} \theta_{0}^{\prime} \\
& +f_{0}^{\prime} \theta_{0}+H^{\prime} f_{0}+H f_{0}^{\prime} .
\end{aligned}
$$

The set of coefficient of $\lambda_{1}^{2}$ :

$$
\begin{aligned}
f_{11}^{\prime \prime \prime} & =-\frac{1}{2} F f_{11}^{\prime \prime}-\frac{5}{2} F^{\prime \prime} f_{11}-\frac{3}{2} f_{1} f_{1}^{\prime \prime}-f_{0} f_{1}^{\prime \prime}+f_{0}^{\prime} f_{1}^{\prime} \\
& \pm \frac{G r_{y}}{R e_{y}^{2}}\left(\theta_{1}^{2}+2 H \theta_{11}\right)+2 F^{\prime} F_{11}^{\prime}+2 f_{1}^{\prime} f_{1}^{\prime}, \\
\frac{1}{P r} \theta_{11}^{\prime \prime} & =-\frac{1}{2} F \theta_{11}^{\prime}-\frac{9}{2} f_{11} H^{\prime}+4 F^{\prime} \theta_{11}-\frac{5}{2} f_{1} \theta_{1}^{\prime} \\
& +f_{0}^{\prime} \theta_{0}+2 f_{1}^{\prime} \theta_{1}-f_{0} \theta_{1}^{\prime} .
\end{aligned}
$$

Expanding Eqs. (19) and (20) using Eqs. (22) and (23) and 
comparing the same powers of $\lambda_{n}(y)$, we get the following forms of boundary conditions for these sets of differential equations of coefficient functions:

$$
\begin{aligned}
& \text { at } \begin{array}{l}
\eta=0: F=0, \quad f_{0}=f_{1}=f_{2}=f_{00}=f_{11}=0 \\
\quad H=1, \theta_{0}=\theta_{1}=\theta_{2}=\theta_{00}=\theta_{11}=0 \\
F^{\prime}=0, f_{0}^{\prime}=f_{1}^{\prime}=f_{2}^{\prime}=f_{00}^{\prime}=f_{11}^{\prime}=0 ;
\end{array} \\
& \text { at } \begin{array}{l}
\eta \rightarrow \infty: F^{\prime}=1, \quad f_{0}^{\prime}=f_{1}^{\prime}=f_{2}^{\prime}=f_{00}^{\prime}=f_{11}^{\prime}=0 \\
\quad H=0, \theta_{0}=\theta_{1}=\theta_{2}=\theta_{00}=\theta_{11}=0 .
\end{array}
\end{aligned}
$$

The afore-mentioned sets of ordinary differential equations are coupled equations. For a given wall temperature variation, these equations are solved using shooting method. The ordinary differential equations are integrated using fourth-order Runge-Kutta method. In the solutions, Prandtl number of water near density inversion is taken to be 11.4. The solutions of the differential equations yield values of coefficient functions with respect to independent variable $\eta$. The solutions of these functions are valid for any arbitrary wall temperature variation. These functions are of general nature, and hence called as 'universal functions'.

For the fluid flow and heat transfer calculations of both aiding and opposing flows, universal functions of stream function and temperature are required along with perturbation variables (see Eqs. (22) and (23)). The universal functions for various $G r_{y} / R e_{y}^{2}$ values are shown in figures 2 and 3 for aiding and opposing flows. Figure 2 shows the universal functions of stream function and temperature for $G r_{y} / R e_{y}^{2}=0$, which corresponds to forced convection extreme. It can be seen from figure 2(a) that the universal functions such as $f_{0}, f_{1}$ and $f_{00}$ come out to be zero for forced convection. This is due to the fact that forced convection flow field remains independent of wall temperature variation. Since perturbation variables $\lambda_{0}, \lambda_{1}, \ldots, \lambda_{00}, \ldots$ depend on wall temperature variation, to get velocity independent of these functions, we need to have $f_{0}, f_{1}$ and $f_{00}$ as zeros from Eq. (22). Hence, the universal functions associated with perturbation variables do not contribute in the calculation of skin friction coefficient for forced convection. Figure 3(a) and 3(b) shows the variation of universal functions of stream function and temperature for aiding and opposing flows, respectively.

\section{Velocity, skin friction coefficient and Nusselt number calculations in terms of universal functions}

The study predicts the velocity and temperature distributions in the boundary layer, drag and heat transfer rates along the wall in mixed convection flows. The temperature distribution in terms of universal functions can be directly calculated using Eq. (23). The velocity distribution, skin friction coefficient and heat transfer rates

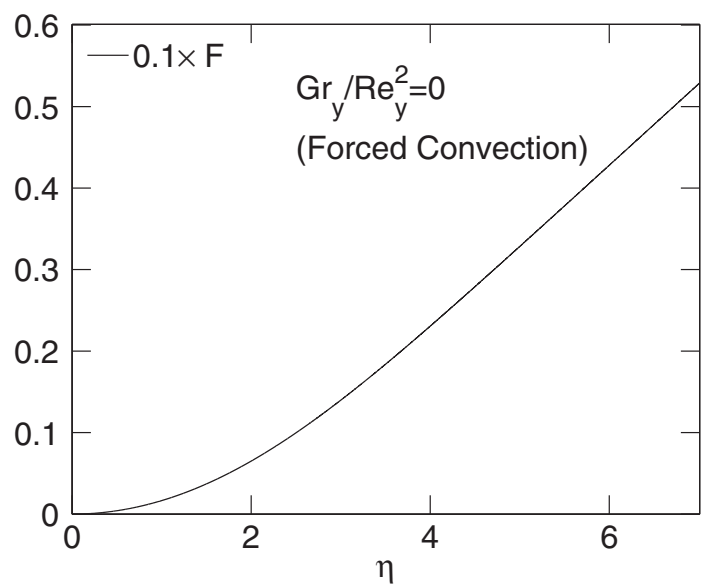

(a) $G r_{y} / R e_{y}^{2}=0$

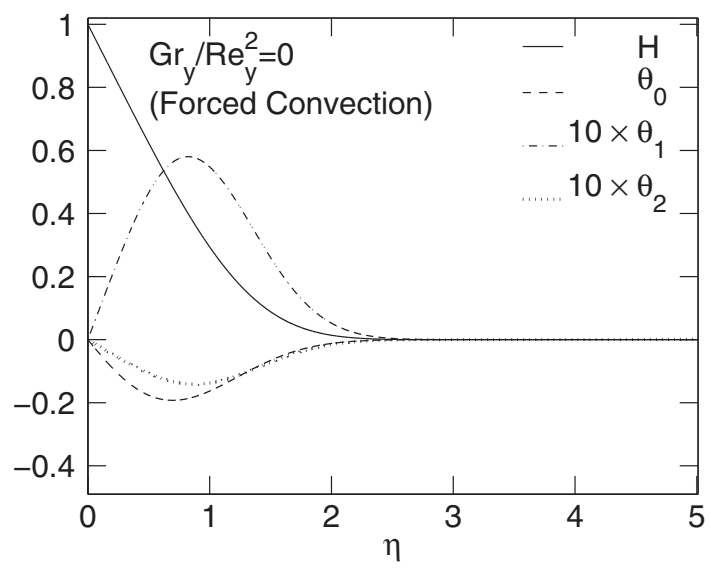

(b) $G r_{y} / R e_{y}^{2}=0$

Figure 2. Universal functions of stream function and temperature for $G r_{y} / R e_{y}^{2}=0$ (forced convection asymptote).

along the wall in terms of universal functions are derived as follows.

In stream function form, the vertical component of velocity can be written as $v=\frac{\partial \psi}{\partial x}$. From this expression, $v$ is obtained, using Eqs. (12) and (14), as follows:

$$
v=\frac{\partial \psi}{\partial x}=\sqrt{v_{\infty} v y} \frac{\partial f}{\partial \eta} \frac{\partial \eta}{\partial x}=v_{\infty} f^{\prime}(\eta) .
$$

Substituting Eq. (22) in this equation, the dimensionless form of vertical component of velocity in terms of universal functions is derived as

$$
\begin{aligned}
\frac{v}{v_{\infty}}=f^{\prime}(\eta)=F^{\prime}(\eta) & +\lambda_{0} f_{0}^{\prime}(\eta)+\lambda_{1} f_{1}^{\prime}(\eta)+\lambda_{2} f_{2}^{\prime}(\eta)+\cdots \\
& +\lambda_{0}^{2} f_{00}^{\prime}(\eta)+\lambda_{1}^{2} f_{11}^{\prime}(\eta)+\cdots
\end{aligned}
$$

Skin friction drag in terms of universal functions is derived here. The local skin friction coefficient can be written as 


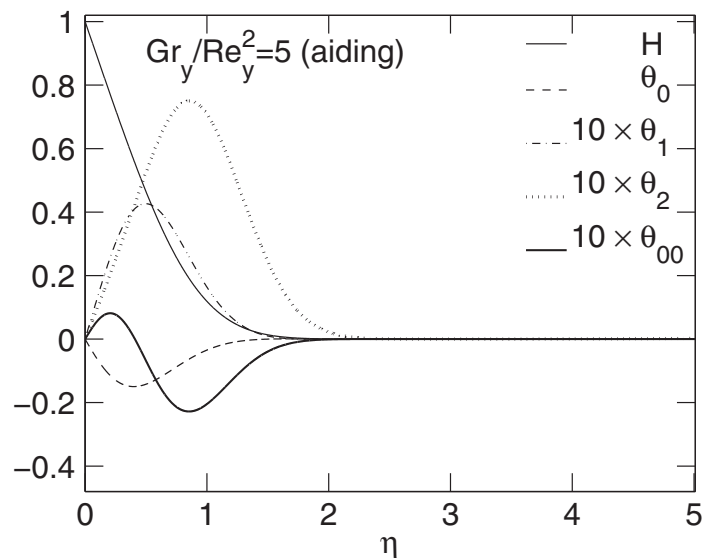

(a) $G r_{y} / R e_{y}^{2}=5$

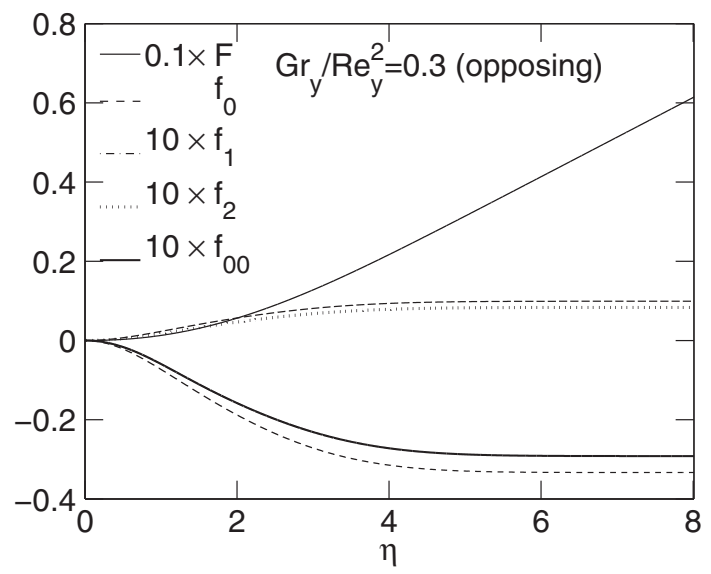

(b) $G r_{y} / R e_{y}^{2}=0.3$

Figure 3. Universal functions of temperature and stream function for aiding and opposing flow.

$$
C_{f y}=\frac{\tau_{\mathrm{w}}}{\rho v_{\infty}^{2}}
$$

where $v_{\infty}$ is free-stream velocity and $\tau_{\mathrm{w}}$ is shear stress at the wall $(x=0)$. Now, the wall shear stress in the form of universal functions becomes

$$
\tau_{\mathrm{w}}=\left.\mu \frac{\partial v}{\partial x}\right|_{x=0}=\left.\mu \frac{\partial}{\partial x}\left(\frac{\partial \psi}{\partial x}\right)\right|_{x=0}=\left.\mu v_{\infty} \sqrt{\frac{v_{\infty}}{v y \partial \eta^{2}} f}\right|_{\eta=0} .
$$

Using Eqs. (36) and (37), we get

$$
\begin{aligned}
\frac{C_{f y}}{R e_{y}^{-1 / 2}}= & \left.f^{\prime \prime}(\eta)\right|_{\eta=0}=F^{\prime \prime}(0)+\lambda_{0} f_{0}^{\prime \prime}(0)+\lambda_{1} f_{1}^{\prime \prime}(0)+ \\
& \lambda_{2} f_{2}^{\prime \prime}(0)+\cdots+\lambda_{0}^{2} f_{00}^{\prime \prime}(0)+\lambda_{1}^{2} f_{11}^{\prime \prime}(0)+\cdots .
\end{aligned}
$$

To calculate the heat transfer rates along the wall, the local heat transfer coefficient $h(y)$ can be written as follows:

$$
h(y)=\frac{q_{w}(y)}{T_{w}-T_{0}} .
$$

The local Nusselt number is given as

$$
N u_{y}=\frac{h(y) y}{k} .
$$

Using Eq. (39) in this equation, the local Nusselt number is

$$
N u_{y}=\frac{q_{w}(y)}{T_{w}-T_{0}} \frac{y}{k} .
$$

From Eq. (13), the temperature difference $\left(T_{w}-T_{0}\right)$ can be given as

$$
T_{w}-T_{0}=\theta_{\eta=0} \frac{q_{w}(y)}{k} y \operatorname{Re}_{y}^{-1 / 2}
$$

where $\theta_{\eta=0}$ is the dimensionless temperature corresponding to the wall $(\eta=0)$. Substituting Eq. (42) in Eq. (41), we get the heat transfer rates as

$$
\frac{N u_{y}}{R e_{y}^{1 / 2}}=\frac{1}{\theta_{\eta=0}} .
$$

Using Eq. (23), the heat transfer rates in Eq. (43) in the form of $N u_{y} / R e_{y}^{1 / 2}$ become

$$
\begin{aligned}
\frac{N u_{y}}{R e_{y}^{1 / 2}}=[H(0) & +\lambda_{0} \theta_{0}(0)+\lambda_{1} \theta_{1}(0)+\lambda_{2} \theta_{2}(0)+\cdots \\
& \left.+\lambda_{0}^{2} \theta_{00}(0)+\lambda_{1}^{2} \theta_{11}(0)+\cdots\right]^{-1} .
\end{aligned}
$$

It can be seen from Eqs. (38) and (44) that $C_{f y} / R e_{y}^{-1 / 2}$ and $N u_{y} / R e_{y}^{1 / 2}$ depend on the universal functions of stream function $\left(F^{\prime \prime}(0), f_{0}^{\prime \prime}(0), f_{1}^{\prime \prime}(0), f_{2}^{\prime \prime}(0), f_{00}^{\prime \prime}(0), f_{11}^{\prime \prime}(0), \ldots\right)$ and temperature $\quad\left(\theta(0), \theta_{0}(0), \theta_{1}(0), \theta_{2}(0), \theta_{00}(0), \theta_{11}(0), \ldots\right)$, respectively, and also on the perturbation variables $\left(\lambda_{0}, \lambda_{1}, \lambda_{2}, \lambda_{0}^{2}, \lambda_{1}^{2}, \ldots\right)$. Since, the universal functions need to be calculated only once for any particular flow, the calculations of skin friction coefficient and heat transfer rates require perturbation variables. As the perturbation variables depend on boundary conditions, for a given wall temperature, the perturbation variables can be calculated. For a particular wall temperature, velocity, temperature, skin friction coefficient and heat transfer rates can be calculated using universal functions and perturbation variables using simple algebraic calculations, without solving any

Table 1. Values of universal constants of stream function in aiding mixed convection flow for various $G r_{y} / R e_{y}^{2}$ values.

\begin{tabular}{lcccccc}
$\frac{G r_{y}}{R e_{y}^{2}}$ & $F^{\prime \prime}(0)$ & \multicolumn{1}{c}{$f_{0}^{\prime \prime}(0)$} & $f_{1}^{\prime \prime}(0)$ & $f_{2}^{\prime \prime}(0)$ & $f_{00}^{\prime \prime}(0)$ & $f_{11}^{\prime \prime}(0)$ \\
\hline 0 & 0.3321 & 0.0000 & 0.0000 & 0.0000 & 0.0000 & 0.0000 \\
0.5 & 0.5366 & -0.0655 & 0.0206 & -0.0051 & 0.0030 & 0.0007 \\
1 & 0.7170 & -0.1162 & 0.0366 & -0.0091 & 0.0032 & 0.0012 \\
5 & 1.8496 & -0.3998 & 0.1253 & -0.0311 & 0.0014 & 0.0038 \\
10 & 2.9790 & -0.6695 & 0.2088 & -0.0518 & 0.0003 & 0.0061 \\
15 & 3.9730 & -0.9045 & 0.2813 & -0.0698 & 0.0001 & 0.0080 \\
\hline
\end{tabular}


Table 2. Values of universal constants of stream function in opposing mixed convection flows for various $G r_{y} / R e_{y}^{2}$ values.

\begin{tabular}{lcccrrr}
\hline$G r_{y}$ & $F^{\prime \prime}(0)$ & $f_{0}^{\prime \prime}(0)$ & $f_{1}^{\prime \prime}(0)$ & $f_{2}^{\prime \prime}(0)$ & \multicolumn{1}{c}{$f_{00}^{\prime \prime}(0)$} & $f_{11}^{\prime \prime}(0)$ \\
\hline$R e_{y}^{2}$ & 0.3321 & 0.0000 & 0.0000 & 0.0000 & 0.0000 & 0.0000 \\
0.05 & 0.3096 & 0.0082 & -0.0026 & -0.0006 & -0.0009 & -0.0001 \\
0.1 & 0.2865 & 0.0170 & -0.0053 & -0.0013 & -0.0021 & -0.0002 \\
0.2 & 0.2385 & 0.0367 & -0.0112 & -0.0027 & -0.0058 & -0.0004 \\
0.3 & 0.1875 & 0.0607 & -0.0183 & -0.0044 & -0.0132 & -0.0006 \\
\hline
\end{tabular}

differential equations. To calculate the skin friction coefficient, the universal functions of stream function at $\eta=0$ for aiding and opposing convection are shown in tables 1 and 2, respectively, for various values of $G r_{y} / R e_{y}^{2}$. For calculation of heat transfer rates, the required universal functions for temperature are given in tables 3 and 4 for aiding and opposing flows, respectively.

\section{Mixed convection flow for power law wall temperature variation}

The universal functions found earlier are valid for any arbitrary wall temperature variation. In this section, the results are applied for power law wall temperature. Power law variation of wall temperature is chosen because many functions can be expressed in terms of power series. Hence, these results are applicable for functions expressible in power series. The wall temperature is taken in the following form:

Table 3. Values of universal constants of temperature in aiding mixed convection flow.

\begin{tabular}{lcccccc}
$\frac{G r_{y}}{R e_{y}^{2}}$ & $H^{\prime}(0)$ & $\theta_{0}^{\prime}(0)$ & $\theta_{1}^{\prime}(0)$ & $\theta_{2}^{\prime}(0)$ & $\theta_{00}^{\prime}(0)$ & $\theta_{11}^{\prime}(0)$ \\
\hline 0 & -0.7609 & -0.4675 & 0.1028 & -0.0228 & 0.0000 & 0.0000 \\
0.5 & -0.8297 & -0.4958 & 0.1055 & -0.0233 & 0.0162 & 0.0004 \\
1 & -0.8816 & -0.5227 & 0.1088 & -0.0239 & 0.0272 & 0.0007 \\
5 & -1.1160 & -0.6628 & 0.1302 & -0.0279 & 0.0635 & 0.0014 \\
10 & -1.2773 & -0.7640 & 0.1473 & -0.0312 & 0.0817 & 0.0019 \\
20 & -1.4810 & -0.8923 & 0.1698 & -0.0357 & 0.1016 & 0.0024 \\
\hline
\end{tabular}

$$
T_{w}-T_{0}=A y^{m}
$$

where $A$ and $m$ are constants. For this power law wall temperature, the variables $\lambda_{0}, \lambda_{1}, \lambda_{0}^{2}, \ldots$ can be calculated using Eq. (21). They can be expressed in terms of $m$ as

$$
\begin{gathered}
\lambda_{0}=m, \lambda_{1}=m(m-1), \lambda_{2}=m(m-1)(m-2), \\
\lambda_{0}^{2}=m^{2}, \lambda_{1}^{2}=m^{2}(m-1)^{2}, \ldots
\end{gathered}
$$

From these expressions, for a given $m$ value, perturbation variables $\left(\lambda_{0}, \lambda_{1}, \lambda_{2}, \lambda_{0}^{2}, \lambda_{1}^{2}, \ldots\right)$ can be calculated. Since the perturbation variables and universal functions are known, velocity, skin friction coefficient and heat transfer rates can be calculated using Eqs. (35), (38) and (44), respectively. It is noticed that calculation of velocity and skin friction coefficient requires derivatives of universal functions for stream function, which come as a solution of system of ordinary differential equations. The temperature variation with respect to $\eta$ is found out using Eq. (23).

\subsection{Validation}

To validate the present methodology, mixed convection boundary layer flow of water over a vertical plate, with density inversion effect, is simulated. The plate is specified with power law varying temperature. The boundary layer flow is solved for various $G r_{y} / R e_{y}^{2}$ and power law index values. The skin friction coefficient for various values of $G r_{y} / R e_{y}^{2}$ and power law index $m$ is shown in table 5. From the table, it can be seen that the present results show good agreement with the results obtained by Vighnesam and Soundalgekar [17].

Table 4. Values of universal constants of temperature in opposing mixed convection flow.

\begin{tabular}{llllrr}
\hline$\frac{G r_{y}}{R e_{y}^{2}}$ & $H^{\prime}(0)$ & $\theta_{0}^{\prime}(0)$ & $\theta_{1}^{\prime}(0)$ & $\theta_{2}^{\prime}(0)$ & $\theta_{00}^{\prime}(0)$ \\
\hline 0 & -0.7609 & -0.4675 & 0.1028 & -0.0228 & 0.0000 \\
0.05 & -0.7524 & -0.4650 & 0.1026 & -0.0228 & -0.0019 \\
0.1 & -0.7436 & -0.4627 & 0.1025 & -0.0227 & -0.0037 \\
0.2 & -0.7244 & -0.4592 & 0.1025 & -0.0227 & -0.0068 \\
0.3 & -0.7026 & -0.4584 & 0.1030 & -0.0227 & -0.00001 \\
\hline
\end{tabular}




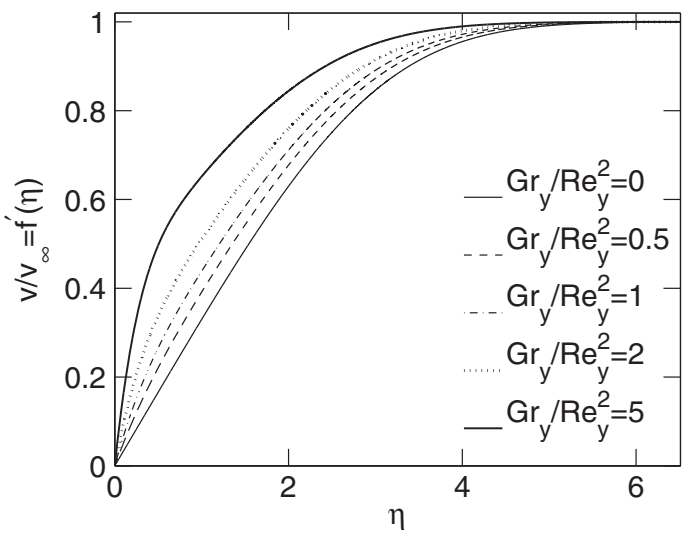

(a) $m=0$

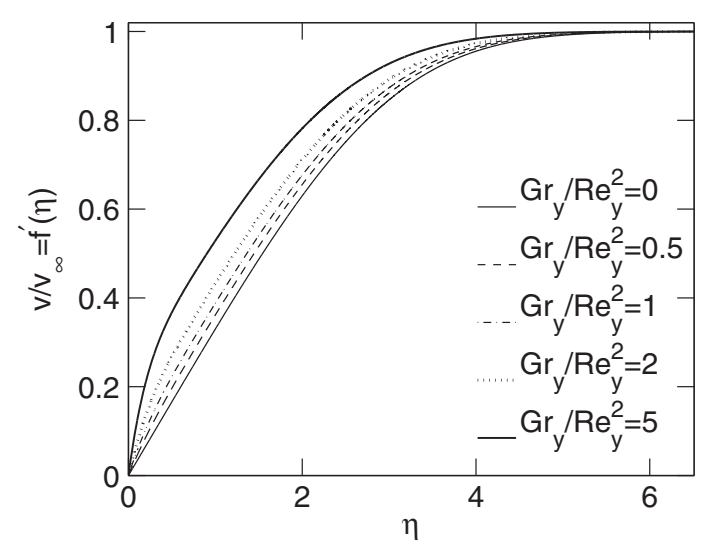

(b) $m=1$

Figure 4. Variation of dimensionless velocity with $\eta$ for various values of $G r_{y} / R e_{y}^{2}$ in aiding flow.

\subsection{Fluid flow solutions}

The dimensionless velocity $\left(v / v_{\infty}=f^{\prime}\right)$ for different $\eta$ can be calculated using Eq. (35). The variation of dimensionless velocity with respect to $\eta$ is shown in figures 4 and 5 for aiding and opposing flows, respectively, for various values of $G r_{y} / R e_{y}^{2}$ and wall temperature power index $m=0$ and $m=1$. It can be seen from the figures that for a given $\eta$ value, the dimensionless velocity increases with $G r_{y} / R e_{y}^{2}$ values in aiding flow, whereas, it decreases in opposing flows, for both $m$ values. This is due to the fact that external and buoyancy forces act in the same direction in aiding flow situation. For a given value of $R e_{y}$, increase in $G r_{y} / R e_{y}^{2}$ represents increase in $G r_{y}$. Increase in $G r_{y}$ value physically represents increase in buoyancy force. Hence, in aiding mixed convection, as the values of $G r_{y} / R e_{y}^{2}$ increase, the strength of fluid flow increases, resulting in increase of dimensionless velocity. However, in opposing flow, the buoyancy forces oppose the external forces. Hence, increased buoyancy forces result in decreased effective driving force. This leads to reduction in dimensionless velocity as the value of $G r_{y} / R e_{y}^{2}$ increases.

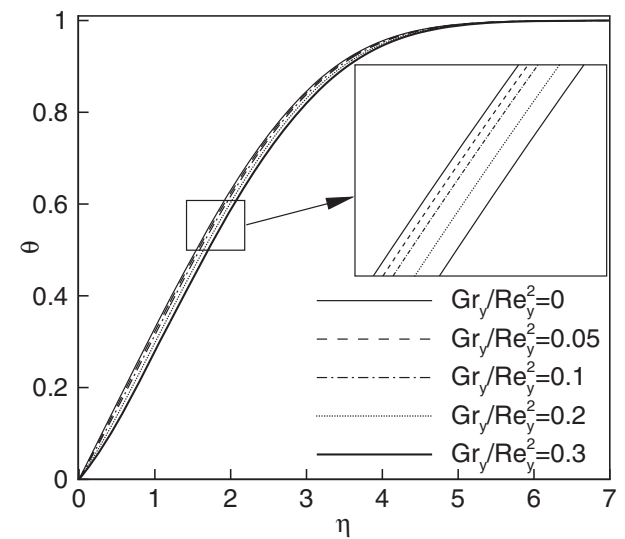

(a) $m=0$

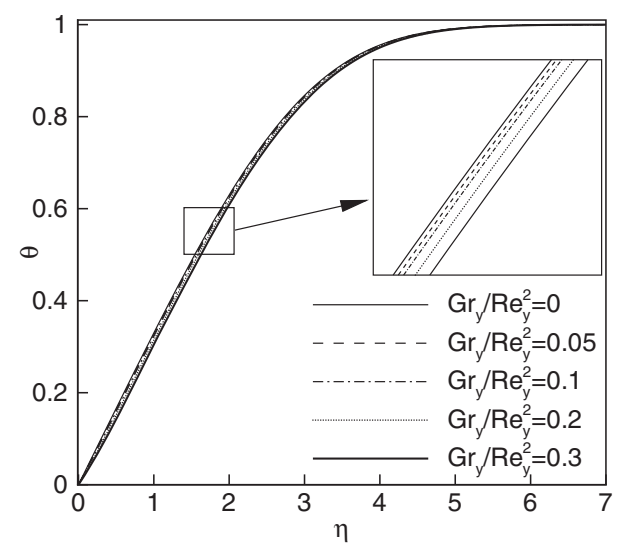

(b) $m=1$

Figure 5. Variation of dimensionless velocity with $\eta$ for various values of $G r_{y} / R e_{y}^{2}$ in opposing flow.

From the variation of dimensionless velocity with respect to $\eta$, we can find out velocity boundary layer thickness. The velocity boundary layer thickness corresponds to $\eta$ at which $v / v_{\infty}$ is equal to 0.99 . The variation of dimensionless velocity boundary layer thickness $\left(\delta_{y}\left[v_{\infty} / v y\right]^{1 / 2}\right)$ with $G r_{y} / R e_{y}^{2}$ for various values of $m$ is shown in figure 6(a) and 6(b), respectively, for aiding and opposing flows. For a given $m$ value, the boundary layer thickness decreases with increasing values of $G r_{y} / R e_{y}^{2}$ in case of aiding mixed convection. This is because convection becomes stronger with increasing $G r_{y} / R e_{y}^{2}$ values, resulting in thinner boundary layer. The opposite trend is observed for opposing flow due to weaker convection as a result of opposing interaction of buoyancy and external forces. It may be noted that dimensionless velocity curves for different $m$ values coincide for $G r_{y} / R e_{y}^{2}=0$ for both aiding and opposing mixed convection. This is due to the fact that $G r_{y} / R e_{y}^{2}=0$ represents forced convection. In forced convection, velocity boundary layer is not affected by the wall temperature boundary condition. Hence, it should be 
Table 5. Comparison of $C_{f y} / R e_{y}^{-1 / 2}$ for power law wall temperature variation obtained in the present study to those presented by Vighnesam and Soundalgekar [17].

\begin{tabular}{rrccr}
\hline$m$ & $\frac{G r_{y}}{R e_{y}^{2}}$ & Present study & Vighnesam and Soundalgekar [17] & 0.03 \\
\hline 0 & 0 & 0.3321 & 0.3320 & 0 \\
0 & -0.5 & 0.0705 & 0.0705 & 0.04 \\
1 & 0.5 & 0.4741 & 0.4743 & 0.02 \\
1 & 0.7 & 0.5272 & 0.5273 & 0.02 \\
\hline
\end{tabular}

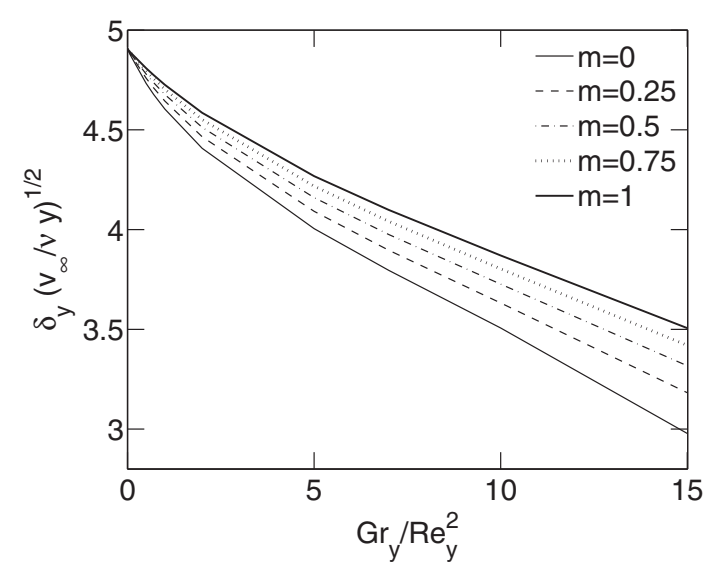

(a) Aiding flow

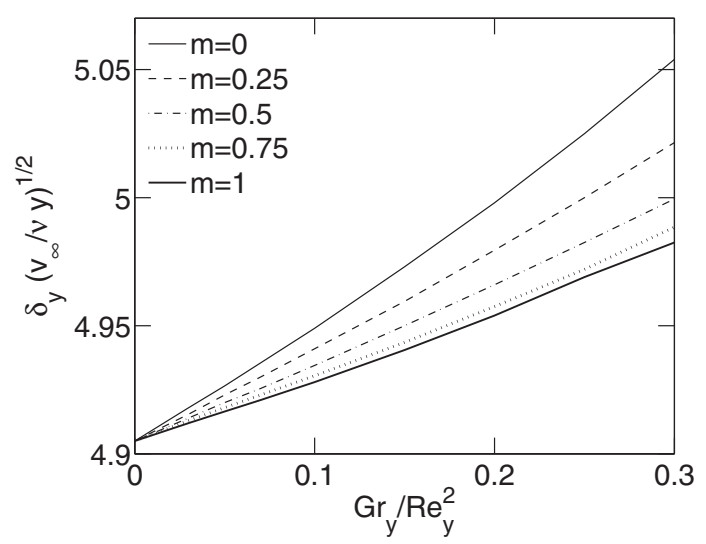

(b) Opposing flow

Figure 6. Variation of velocity boundary layer thickness $\left(\delta_{y}\left[v_{\infty} / v y\right]^{1 / 2}\right)$ with $G r_{y} / R e_{y}^{2}$ for various $m$ values.

independent of $m$ values. Hence, for $G r_{y} / R e_{y}^{2}=0$, boundary layer thickness becomes the same for all $m$ values.

The variation of skin friction coefficient $\left(C_{f y} / R e_{y}^{-1 / 2}\right)$ with $G r_{y} / R e_{y}^{2}$ values is shown in figure 7(a) and 7(b) for aiding and opposing flows, respectively, for various $m$ values. In both of these flows, $C_{f y} / R e_{y}^{-1 / 2}$ values start with forced convection values and vary towards natural convection asymptote with increasing values of $G r_{y} / R e_{y}^{2}$ for all power-index values. For a given $m$ value, value of skin friction coefficient is greater than corresponding forced convection values in aiding mixed convection and

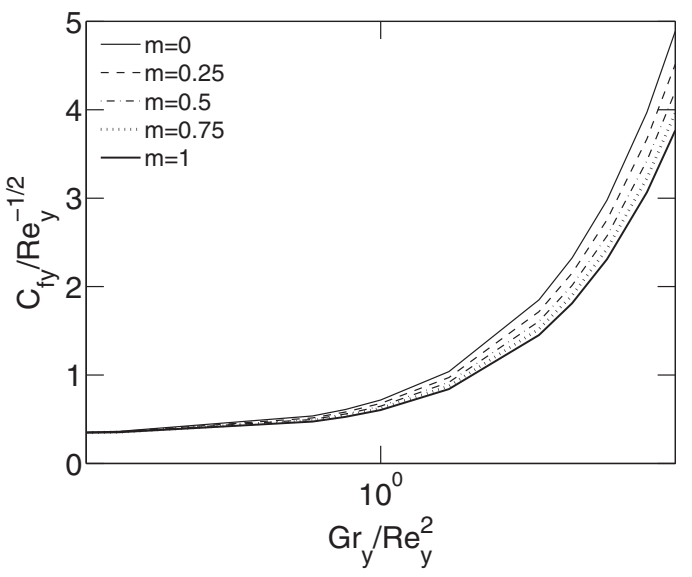

(a) Aiding flow

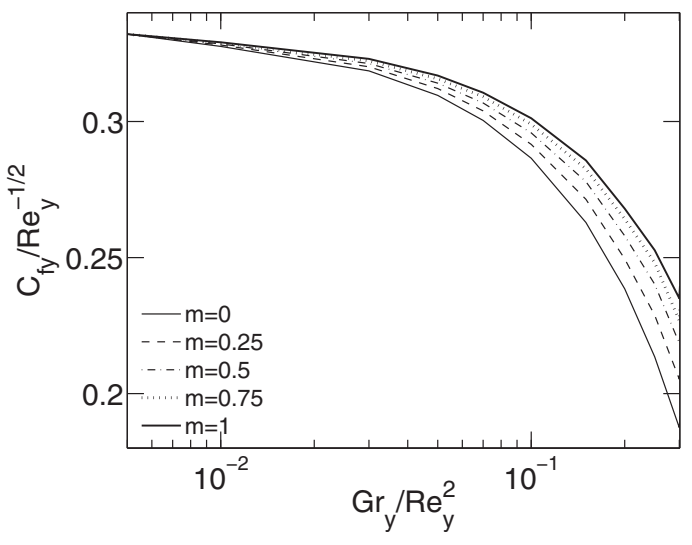

(b) Opposing flow

Figure 7. Variation of skin friction coefficient with $G r_{y} / R e_{y}^{2}$ for various power index values $m$.

$C_{f y} / R e_{y}^{-1 / 2}$ increases with increasing $G r_{y} / R e_{y}^{2}$ values because buoyancy and external forces act in the same direction in aiding flow. Here, higher the value of $G r_{y} / R e_{y}^{2}$, stronger the flow. Due to increased flow strength, the shear stress on the wall increases due to increased velocity gradient on plate. Hence, skin friction coefficient increases with increasing $G r_{y} / R e_{y}^{2}$ values.

In opposing flow, the value of skin friction coefficient is lower than corresponding forced convection values and it decreases with increasing $G r_{y} / R e_{y}^{2}$ values. This trend is opposite to the trend in aiding mixed convection. This is 


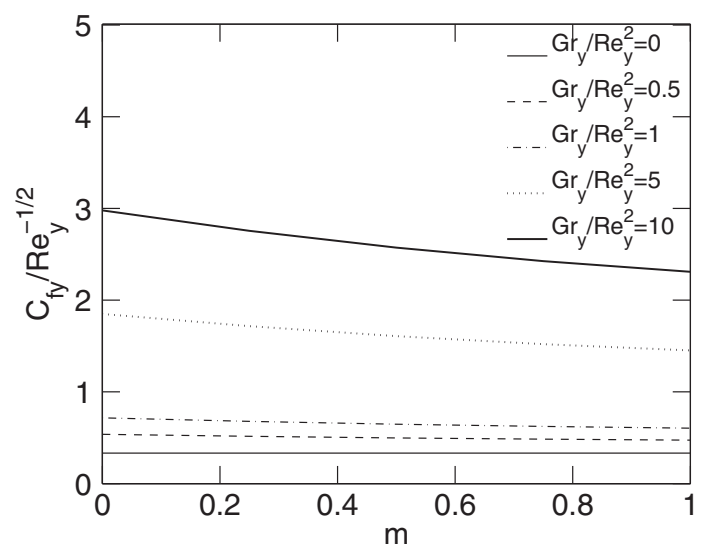

(a) Aiding flow

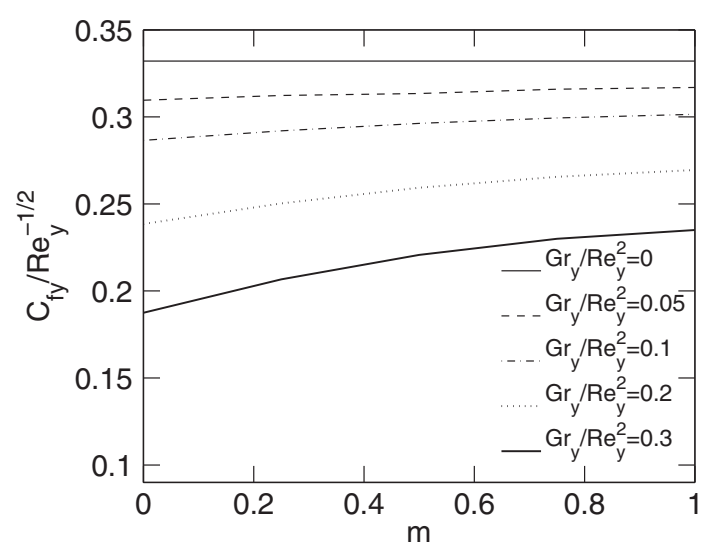

(b) Opposing flow

Figure 8. Variation of skin friction coefficient with $m$ for various $G r_{y} / R e_{y}^{2}$ values.

due to the opposite nature of interaction of buoyancy and external forces in opposing flows.

The variation of $C_{f y} / R e_{y}^{-1 / 2}$ with wall temperature power index for various $G r_{y} / R e_{y}^{2}$ values is shown in figure 8(a) and 8(b) for aiding and opposing mixed convection, respectively. These figures show that for a particular value of $G r_{y} / R e_{y}^{2}$, skin friction coefficient decreases with increasing value of power index $m$ in aiding flow. However, the skin friction coefficient increases with increasing value of $m$ in opposing flow. In the figures, plot for $G r_{y} / R e_{y}^{2}=0$ corresponds to forced convection in both aiding and opposing flows. It can also be observed that for $G r_{y} / R e_{y}^{2}=0$, the value of skin friction coefficient remains constant with increasing $m$ values because forced convection flow is independent of the wall temperature boundary condition.

\subsection{Heat transfer solutions}

The variation of dimensionless temperature with $\eta$ is presented for $m=0$ and $m=1$, which is shown in figures 9

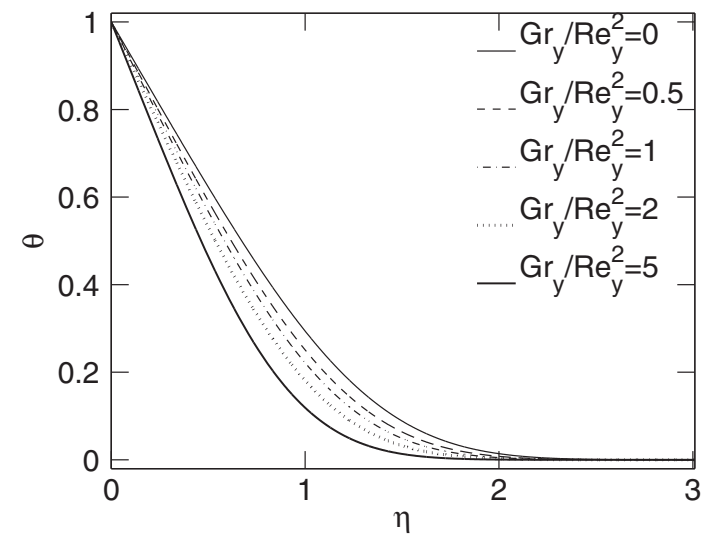

(a) $m=0$

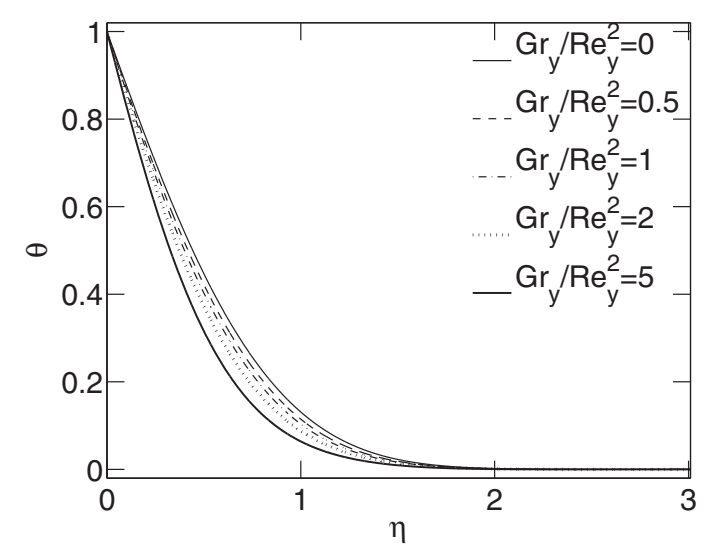

(b) $m=1$

Figure 9. Variation of dimensionless temperature with $\eta$ for various values of $G r_{y} / R e_{y}^{2}$ in aiding flow.

and 10 for aiding and opposing mixed convection, respectively, for various $G r_{y} / R e_{y}^{2}$ values. It is observed from the figures that at a particular $\eta$ value, the dimensionless temperature decreases with increasing values of $G r_{y} / R e_{y}^{2}$ for aiding flow, while the reverse trend is observed for opposing flow. As increased $G r_{y} / R e_{y}^{2}$ represents resulting strengthened convection in aiding flow, it leads to lowered temperatures. However, in opposing flow, increasing $G r_{y} / R e_{y}^{2}$ represents weakened convection, leading to higher temperatures.

From the computations of $\theta$ variations with $\eta$, the thermal boundary layer thickness can be found out. Thermal boundary layer thickness corresponds to horizontal distance from wall to a point in boundary layer at which $\theta$ value becomes 0.01 . This means that the boundary layer thickness corresponds to $\eta$ value at which $\theta$ value becomes 0.01 . The variation of dimensionless boundary layer thickness $\delta_{T}\left(v_{\infty} / v y\right)^{1 / 2}$ with $G r_{y} / R e_{y}^{2}$ for various values of powerindex $m$ is shown in figure 11 for both cases of aiding and opposing mixed convection. It can be observed from this 


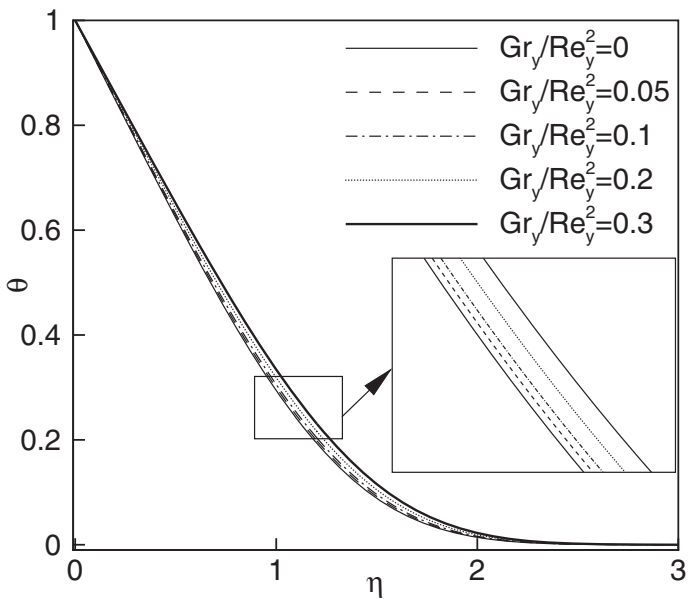

(a) $m=0$

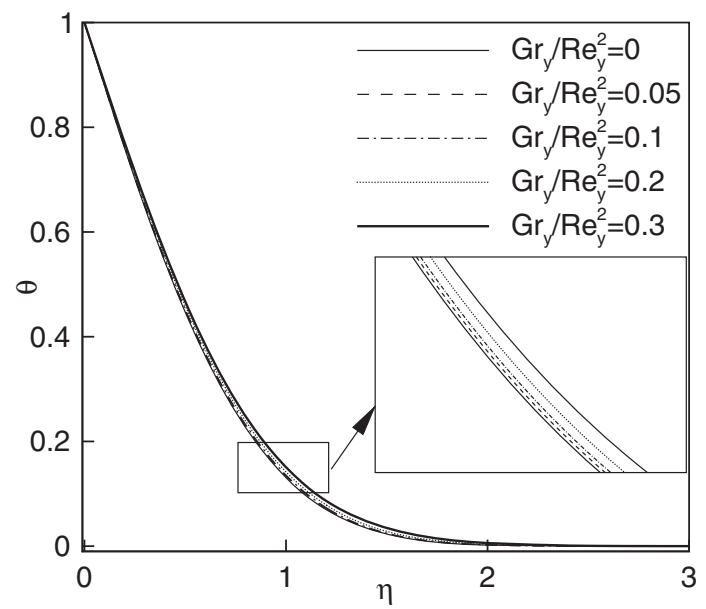

(b) $m=1$

Figure 10. Variation of dimensionless temperature with $\eta$ for various values of $G r_{y} / R e_{y}^{2}$ in opposing flow.

figure that boundary layer thickness, at a given $y$ value, decreases monotonically with increasing $G r_{y} / R e_{y}^{2}$ values for aiding mixed convection. In aiding flow, as buoyancy complement external forces, increasing $G r_{y} / R e_{y}^{2}$ value results in stronger convection and thinner boundary layer. The opposite trend is observed for the case of opposing flow because, in this case, buoyancy opposes external forces.

To see the effect of $G r_{y} / R e_{y}^{2}$ values on heat rates, the variation of $N u_{y} / R e_{y}^{1 / 2}$ with $G r_{y} / R e_{y}^{2}$ values for aiding case is shown in figure 12(a), for various $m$ values. The corresponding plots for opposing mixed convection are shown in figure 12(b). In aiding flow case, for a given $m$ value, heat rates increase with increasing $G r_{y} / R e_{y}^{2}$ values. However, the heat rates decrease with values of $G r_{y} / R e_{y}^{2}$ in opposing flow case. In these results, $N u_{y} / R e_{y}^{1 / 2}$ values for $G r_{y} / R e_{y}^{2}=$ 0 correspond to forced convection asymptote. The Nusselt number starts with forced convection values and varies towards natural convection asymptote with increasing

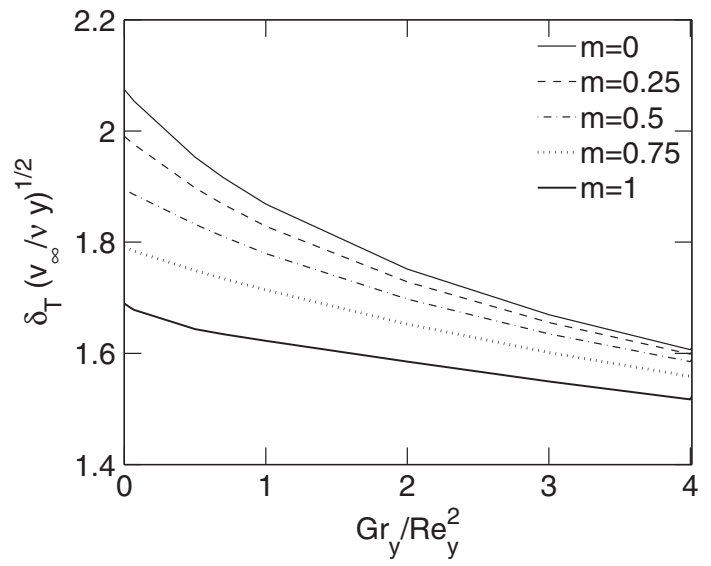

(a) Aiding flow

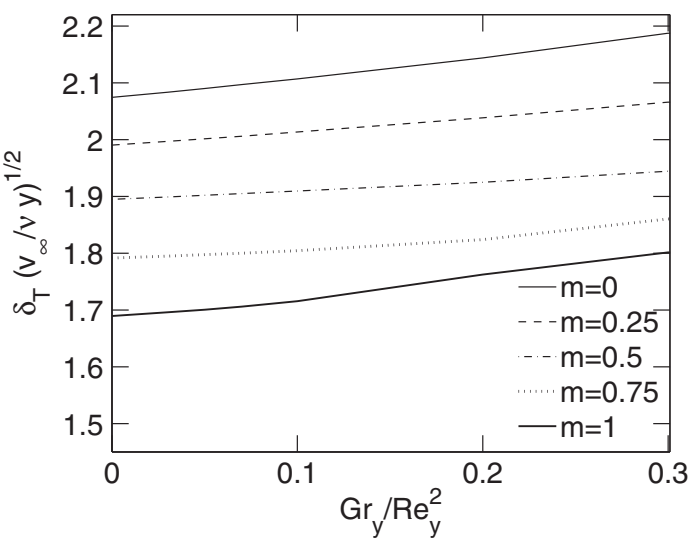

(b) Opposing flow

Figure 11. Variation of thermal boundary layer thickness $\left(\delta_{T}\left[v_{\infty} / v y\right]^{1 / 2}\right)$ with $G r_{y} / R e_{y}^{2}$ for various $m$ values.

$G r_{y} / R e_{y}^{2}$. When mixed convection approaches forced convection extreme, the variation of Nusselt number becomes constant. This is typical in mixed convection flows. It can be seen from the figures that for a given value of $R e_{y}$, Nusselt number increases with Grashof number. This is due to the fact that increased Grashof number represents higher buoyancy forces, and hence higher heat transfer rates. For a given set of $G r_{y}$ and $R e_{y}$ values, the Nusselt numbers in case of aiding mixed convection are higher than those of opposing flow. This is because the external flow complements buoyancy forces in aiding flow. However, in opposing flow, the external forces oppose buoyancy forces, resulting in lower heat transfer rates. For opposing mixed convection, solutions are obtained for lower values of $G r_{y} / R e_{y}^{2}$, because at higher values, flow separation takes place, for which boundary layer approximations are not applicable as the boundary layer tends to separate.

The variation of heat transfer rates in terms of $N u_{y} / R e_{y}^{1 / 2}$ with variation of power index value $m$ is shown in figure 13(a) 


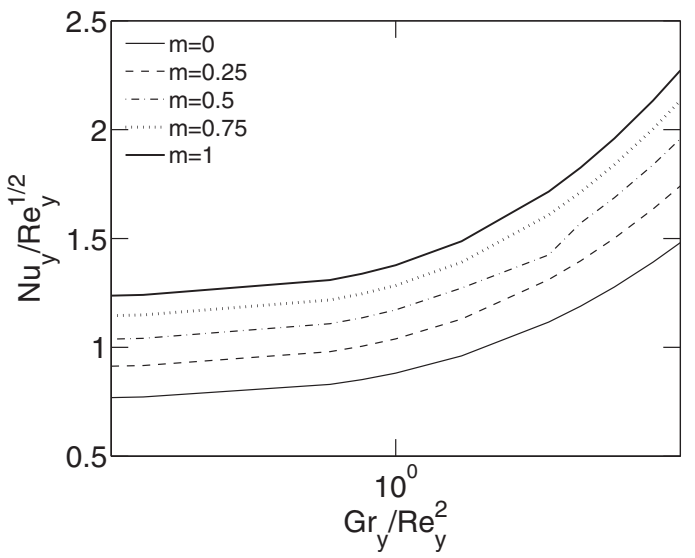

(a) Aiding flow

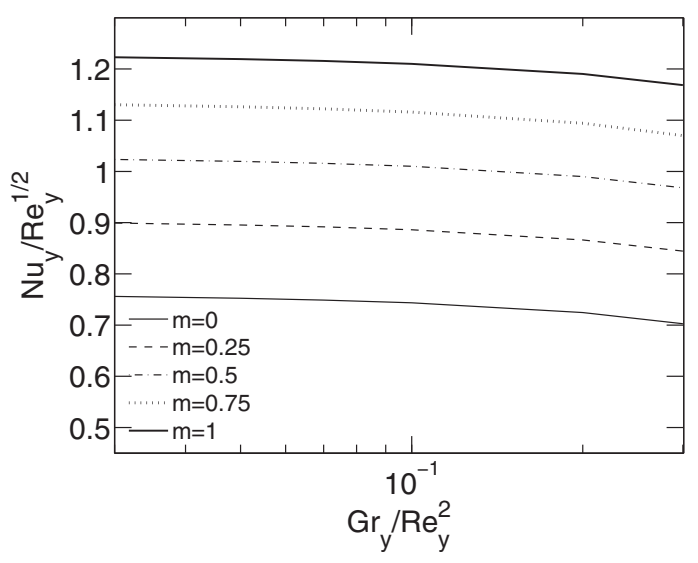

(b) Opposing flow

Figure 12. Variation of $N u_{y} / R e_{y}^{1 / 2}$ with $G r_{y} / R e_{y}^{2}$ for various values of $m$.

and 13(b), respectively, for aiding and opposing cases for various $G r_{y} / R e_{y}^{2}$ values. From these figures, it is observed that the heat transfer rates increase with power index $m$ for a given $G r_{y} / R e_{y}^{2}$ value for both aiding and opposing flow situations. This is because with increasing $m$ value, temperatures increase at all points on the plate. Hence, at any given location of the plate, the temperature difference between the plate and fluid increases with increasing $m$ for any given $G r_{y} / R e_{y}^{2}$ value, resulting in higher heat transfer rates. This is the reason why heat transfer rates increase with increasing $m$ values for all $G r_{y} / R e_{y}^{2}$ values in both the cases of aiding and opposing flows.

The essence of the perturbation method used in the present study is that these results can be readily used for any arbitrary wall temperature variation without the need for solving any differential equation. For different temperature variations, $\lambda_{0}, \lambda_{1}, \lambda_{2}, \lambda_{0}^{2}, \lambda_{1}^{2}, \ldots$ need to be calculated from Eq. (46), which involves simple algebraic calculations.

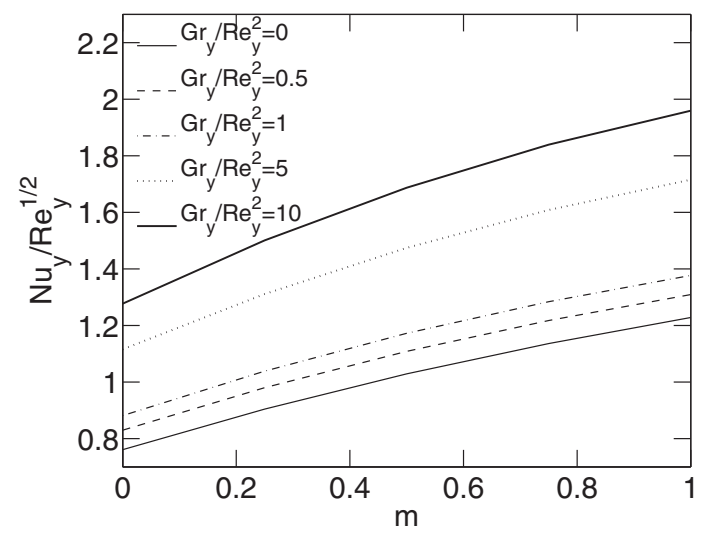

(a) Aiding flow

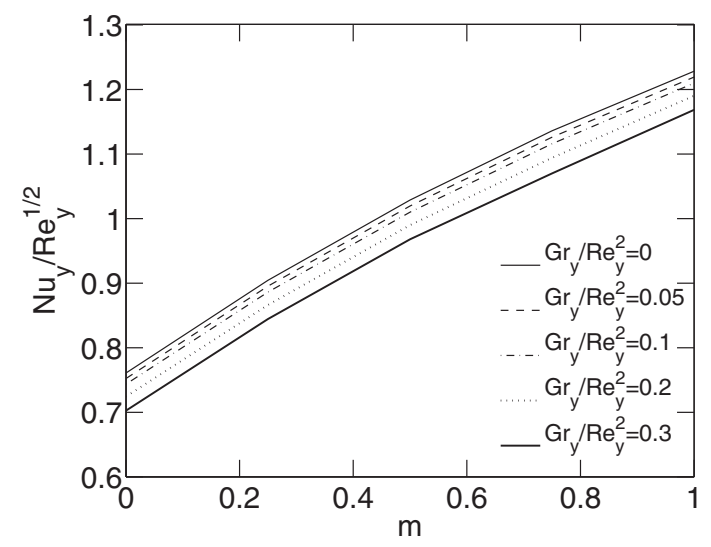

(b) Opposing flow

Figure 13. Variation of $N u_{y} / R e_{y}^{1 / 2}$ with $m$ for various values of $G r_{y} / R e_{y}^{2}$.

\section{Conclusion}

Mixed convection flow over a vertical surface with varying wall temperature is studied using perturbation method. The wall temperature differentials are taken as perturbation functions. The sets of coupled differential equations are solved to find out universal functions for stream function and temperature, which are independent of any arbitrarily varying wall temperature. Power law variation for wall temperature is taken to demonstrate the universal applicability of universal functions. Results are presented for velocity and temperature distributions in boundary layer, velocity and thermal boundary layer thicknesses, skin friction coefficient and heat transfer rates along the plate for various values of $G r_{y} / R e_{y}^{2}$ and wall temperature power law index. It is found that heat transfer rates increase linearly with increasing value of power law index for both aiding and opposing flows, for all $G r_{y} / R e_{y}^{2}$ values, for the range of $G r_{y} / R e_{y}^{2}$ values considered in the study. The main advantage of the perturbation 
method is that the obtained universal functions are valid for any arbitrary temperature variation. Hence, for different boundary conditions, solutions can be obtained by calculating only perturbation variables along with simple algebraic calculations. We do not need to solve any differential equation additionally to get the solutions.

\section{Nomenclature}

A

$C_{f}$

$f$

$F, f_{0}, f_{1}, \ldots$,

$f_{00}, f_{11}(\eta), \ldots$

$g$

$G r$

$h$

$H, \theta_{0}, \theta_{1}, \ldots$, $\theta_{00}, \theta_{11}(\eta), \ldots$

$k$

$m$

$\mathrm{Nu}$

$\operatorname{Pr}$

$R e$

$T$

$T_{0}$

$u, v$

$x, y$

constant in power law wall temperature

variation (Eq. (45))

skin friction coefficient

non-dimensional stream function

universal functions for velocity in

perturbation technique

gravitational acceleration $\left(\mathrm{m} / \mathrm{s}^{2}\right)$

modified Grashof number

heat transfer coefficient $\left(\mathrm{W} / \mathrm{m}^{2} \mathrm{~K}\right)$

universal functions for temperature

in perturbation technique

thermal conductivity of fluid (W/m K)

power index of power law variation of wall temperature

Nusselt number

Prandtl number

Reynolds number

temperature $\left({ }^{\circ} \mathrm{C}\right)$

temperature at maximum density of water $\left({ }^{\circ} \mathrm{C}\right)$

velocity components in $x$ - and $y$-direction,

respectively $(\mathrm{m} / \mathrm{s})$

Cartesian coordinates (m)

\section{Greek Symbols}

$\delta_{v}$

$\delta_{T}$

$\eta$

$\gamma$

$\lambda_{0}, \lambda_{1}, \ldots, \lambda_{n}$ a set of variables that are functions

of $y$

$\mu$

$v$

$\psi$

$\rho$

$\rho_{0}$

$\tau$

$\theta$

thermal diffusivity $\left(\mathrm{m}^{2} / \mathrm{s}\right)$

velocity boundary layer thickness (m)

thermal boundary layer thickness (m)

non-dimensional independent variable

constant in function (Eq. (1)) of density

variation of water with temperature

$\left({ }^{\circ} \mathrm{C}^{-2}\right)$

dynamic viscosity $\left(\mathrm{Ns} / \mathrm{m}^{2}\right)$

kinematic viscosity $\left(\mathrm{m}^{2} / \mathrm{s}\right)$

dimensional stream function $\left(\mathrm{m}^{2} / \mathrm{s}\right)$

density $(1 /$ specific volume $)\left(\mathrm{kg} / \mathrm{m}^{3}\right)$

maximum density of water $\left(\mathrm{kg} / \mathrm{m}^{3}\right)$

shear stress $\left(\mathrm{N} / \mathrm{m}^{2}\right)$

non-dimensional temperature

\author{
Subscripts \\ w wall \\ y local quantity \\ $\infty$ free-stream quantity
}

\section{References}

[1] Lloyd J R and Sparrow E M 1970 Combined forced and free convection flow on vertical surfaces. Int. J. Heat Mass Transfer 13: 434-438

[2] Acrivos A 1966 On the combined effect of forced and free convection heat transfer in laminar boundary layer flows. Chem. Eng. Sci. 21(4): 343-352

[3] Szewczyk A A 1964 Combined forced and free-convection laminar flow. J. Heat Transfer-Trans. ASME 86(4): 501-507

[4] Hussain T and Afzal N 1988 Computer extension of perturbation series for mixed convection on a vertical plate: favourable and adverse flows. Fluid Dyn. Res. 4(2): 107

[5] Lin H T and Chen C C 1988 Mixed convection on vertical plate for fluids of any Prandtl number. Int. J. Heat Mass Transfer 22: 159-168

[6] Lin H T and Hoh H L 1997 Mixed convection from an isothermal vertical flat plate moving in parallel or reversely to a free stream. Heat Mass Transfer 32: 441-445

[7] Raju M S, Liu X Q and Law C W 1984 A formulation of combined forced and free convection past horizontal and vertical surfaces. Int. J. Heat Mass Transfer 27: 2215-2224

[8] Chen T S, Armaly B F and Ramachandran N 1986 Correlations for laminar mixed convection flows on vertical, inclined and horizontal flat plates. J. Heat Transfer 108: 835-840

[9] Merkin J H and Pop I 2002 Mixed convection along a vertical surface: similarity solutions for uniform flow. Fluid Dyn. Res. 30: 233-250

[10] Saeid N H 2005 Mixed convection flow along a vertical plate subjected to time-periodic surface temperature oscillations. Int. J. Therm. Sci. 44: 531-539

[11] Ishak A, Nazar R and Pop I 2008 Mixed convection boundary layer flow adjacent to a vertical surface embedded in a stable stratified medium. Int. J. Heat Mass Transfer 51: 3693-3695

[12] Gryzagoridis J 1975 Combined free and forced convection from an isothermal vertical plate. Int. J. Heat Mass Transfer 18: 911-916

[13] Kobus C J and Wedekind G L 1996 Modeling the local and average heat transfer coefficient for an isothermal vertical flat plate with assisting and opposing combined forced and natural convection. Int. J. Heat Mass Transfer 39(13): 2723-2733

[14] Venugopal G, Balaji C and Venkateshan S P 2008 A correlation for laminar mixed convection from vertical plates using transient experiments. Int. J. Heat Mass Transfer 44: $1417-1425$

[15] Goren S L 1966 On free convection in water at $4{ }^{\circ} \mathrm{C}$. Chem. Eng. Sci. 21: 515-518

[16] Gebhart B, Bendell M S and Shaukatullah H 1978 Buoyancy induced flows adjacent to horizontal surfaces in water near its density extremum. Int. J. Heat Mass Transfer 22: 137-149

[17] Vighnesam N V and Soundalgekar V M 1997 Combined free and forced convection flow of water at $4{ }^{\circ} \mathrm{C}$ from a vertical plate with variable temperature. Indian J. Eng. Mater. Sci. 5: 124-126 
[18] Kumaran V and Pop I 2006 Steady free convection boundary layer over a vertical flat plate embedded in a porous medium filled with water at $4{ }^{\circ} \mathrm{C}$. Int. J. Heat Mass Transfer 49: 3240-3252

[19] Chin K E, Nazar R, Arifin N M and Pop I 2007 Effect of variable viscosity on mixed convection boundary layer flow over a vertical surface embedded in a porous medium. Int. Commun. Heat Mass Transfer 34: 464-473

[20] Sparrow E M and Yu H S 1971 Local non-similarity thermal boundary-layer solutions. J. Heat Transfer-Trans. ASME 93: $328-334$

[21] Kao K T 1976 Locally non-similar solution for laminar free convection adjacent to a vertical wall. J. Heat Transfer 98: 321-322

[22] Kelleher M and Yang K T 1972 A Gortler-type series for laminar free convection along a non-isothermal vertical plate. Quart. J. Mech. Appl. Math. 25: 445-457
[23] Yang K T and Jerger E W 1964 First-order perturbations of laminar free-convection boundary layers on a vertical plate. J. Heat Transfer 86(1): 107-114

[24] Seetharamu K N and Dutta P 1988 Mixed convection about a non-isothermal vertical surface in a porous medium. Int. J. Numer. Methods Fluids 8(6): 723-735

[25] Gavara M R, Dutta P and Seetharamu K N 2012 Mixed convection adjacent to non-isothermal vertical surfaces. Int. J. Heat Mass Transfer 55(17-18): 4580-4587

[26] Tong W and Koster J N 1993 Natural convection of water in a rectangular cavity including density inversion. Int. J. Heat Fluid Flow 14(4): 366-375

[27] Schlichting H and Gersten K 2014 Boundary layer theory, 8th revised ed. Springers

[28] Van Dyke M D 1975 Perturbation methods in fluid mechanics. New York: Parabolic Press 